\title{
DETECTION OF GRAVITATIONAL RADIATION
}

\author{
J. A. TYSON
}

Bell Laboratories, Murray Hill, N.J.07974, U.S.A.

\begin{abstract}
The claims by $\mathrm{J}$. Weber must be examined in the context of system time-response and signalto-noise ratio. After briefly discussing the sources and spectral distribution of system noise, we discuss optimal time-domain filtering. Although five independent groups have searched for two-antenna coincidences, only Weber has claimed any excess at zero time lag. We present preliminary results of a high sensitivity two-antenna coincidence search.
\end{abstract}

In this talk I will attempt to review the problem of maximizing the sensitivity of Weber-type gravitational antennas, and I will give a summary of the latest searches for gravitational radiation (GR). The techniques of GR detection have reached such a point that the next generation of antennas may be sufficiently sensitive to GR that we can envision all sky surveys and searches for GR from likely astrophysical sources. In this new field we are always reminded of the pioneering efforts of Joseph Weber. Without his continuing work we would not be here today discussing GR as a possible experimental reality.

Weber's claimed observation (Weber 1969, 1970a, b, 1972) of intensive bursts of kilohertz-band GR is based on two significant features in his data: (1) an excess number of coincidences above chance between two antennas, and (2) a sidereal correlation of these coincidence events. I will discuss here only tests to check on his claim (1) of a statistically significant excess at zero time lag. After reviewing the detection sensitivity limits set by extraneous system noise, I will discuss the limits presently set by several independent experiments, with emphasis on a two-antenna coincidence experiment at Bell Labs and the University of Rochester.

As the gravitational wave passes by an elastic solid, the wave does work against the electrical forces in the solid and, in the case of a Weber-type antenna, the aluminum bar absorbs some energy from the wave. This energy absorption cross section is proportional to the mass of the bar multiplied by $(v / c)^{2}$, where $v$ is the sound velocity in the bar. Obviously, we could significantly improve the absorption cross section by fabricating the antenna from solid nuclear matter. But adequately instrumenting a neturon star would seem an impossible task. More exactly, for a geometrically linear antenna the gravitational wave appears as an acceleration gradient along the axis of the antenna, thereby coupling only to the odd longitudinal elastic modes of oscillation. For a bar geometry and in the case of a broadband source spectrum (a short burst of GR), the integrated absorption cross section is given by

$$
\int \sigma(v) \mathrm{d} v=2 G m \omega^{2} l^{2} / \pi^{3} c^{3} n^{2}
$$

where we have substituted the dispersion relation for a bar of length $l$, longitudinal resonance frequency $\omega$, and mode number $n$. This absorbed energy will produce an 
observable change in the bar energy if the obscuring noise is comparatively small. We must study how the various sources of extraneous noise may be minimized.

We wish to convert the bar oscillations into an electrical signal, adding as little noise as possible. If the elastic potential energy in an odd longitudinal mode of the bar is measured by attaching electromechanical transducers symmetrically around the center of the bar, the electrical equivalent circuit appears as in Figure 1. The narrow mechanical resonance of the bar is represented by the electrical resonance of $L_{1} C_{1} R_{1}$. The electromechanical transducer of capacitance $C_{2}$ electromechanical efficiency

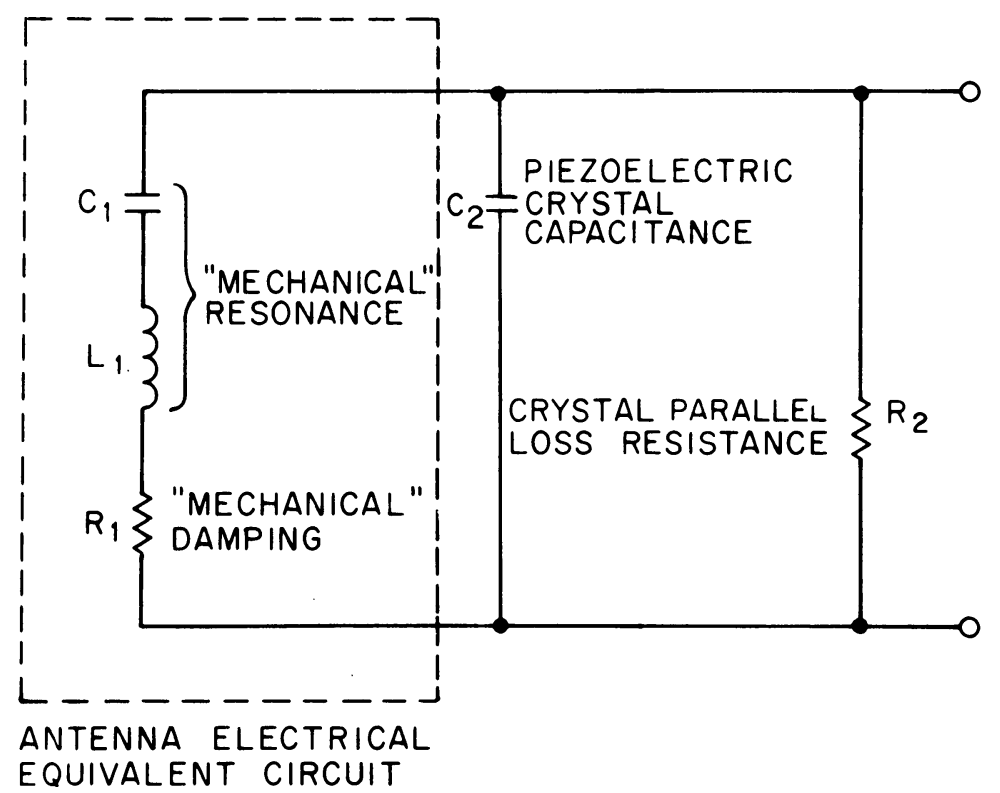

Fig. 1. The equivalent electrical circuit of the antenna, looking into the transducer terminals. The voltage available is a fraction $\beta=C_{1} / C_{2}$ of the voltage across $C_{1}$, representing the electromechanical efficiency $\beta$.

$\beta=C_{1} / C_{2}$, and parallel loss $R_{2}$ creates the electrical impedance of the antenna as seen across the two terminals on the right. The narrowband noise due to the Johnson noise of $R_{1}$ filtering through the antenna impedance is the Brownian motion of the bar in this single mode. Small changes in the bar energy are masked by both this Brownian motion and also the wideband noise from the transducer loss $R_{2}$. In addition to these two fundamental noise sources, we must contend with the additional series and parallel noises of the preamplifier, as shown in Figure 2. The wideband noise from $R_{s}$ is the limiting noise in all the present experiments searching for GR. This is because we expect a sudden change in bar energy upon reception of a burst of GR, and therefore we must have a wide electronics bandwidth in order to optimize detection of this kind of signature.

The spectral distribution of noise coming from the antenna and first amplifier is sketched in Figure 3. The occurrance of a detected GR burst would appear as a brief 


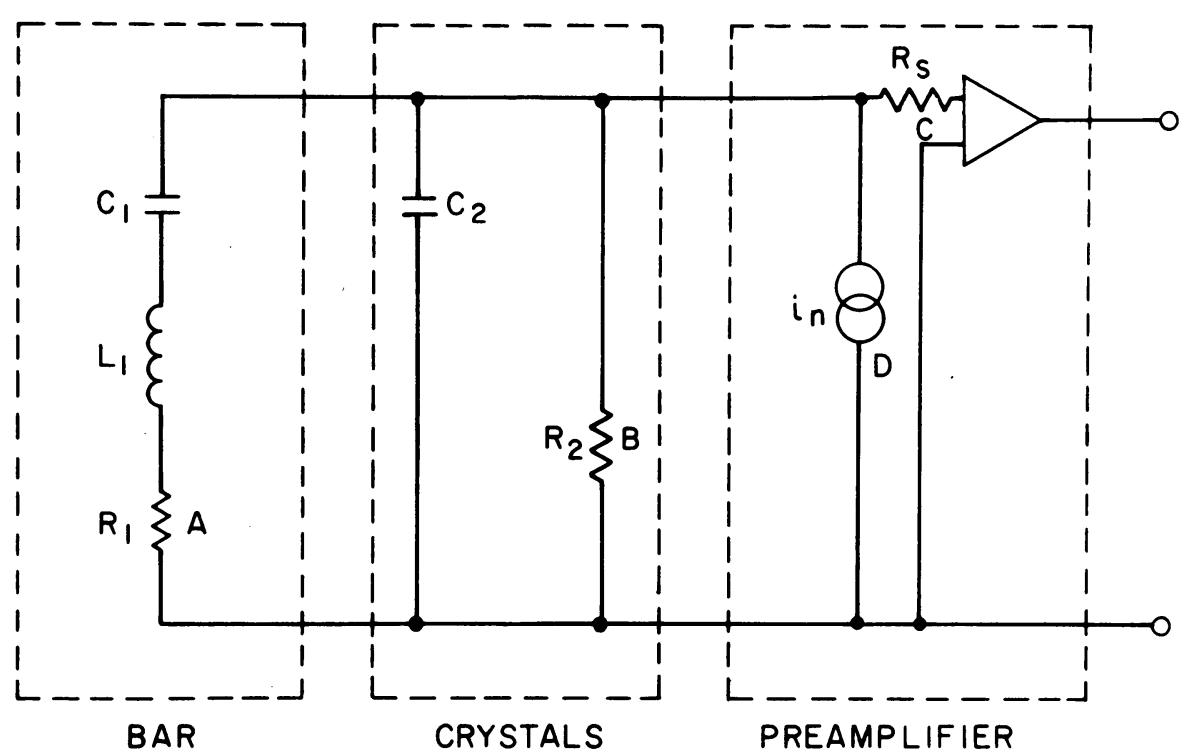

Fig. 2. The four irreducible noise sources $\left(R_{1}, R_{2}, R_{s}, i_{n}\right)$ are shown in this equivalent input circuit.

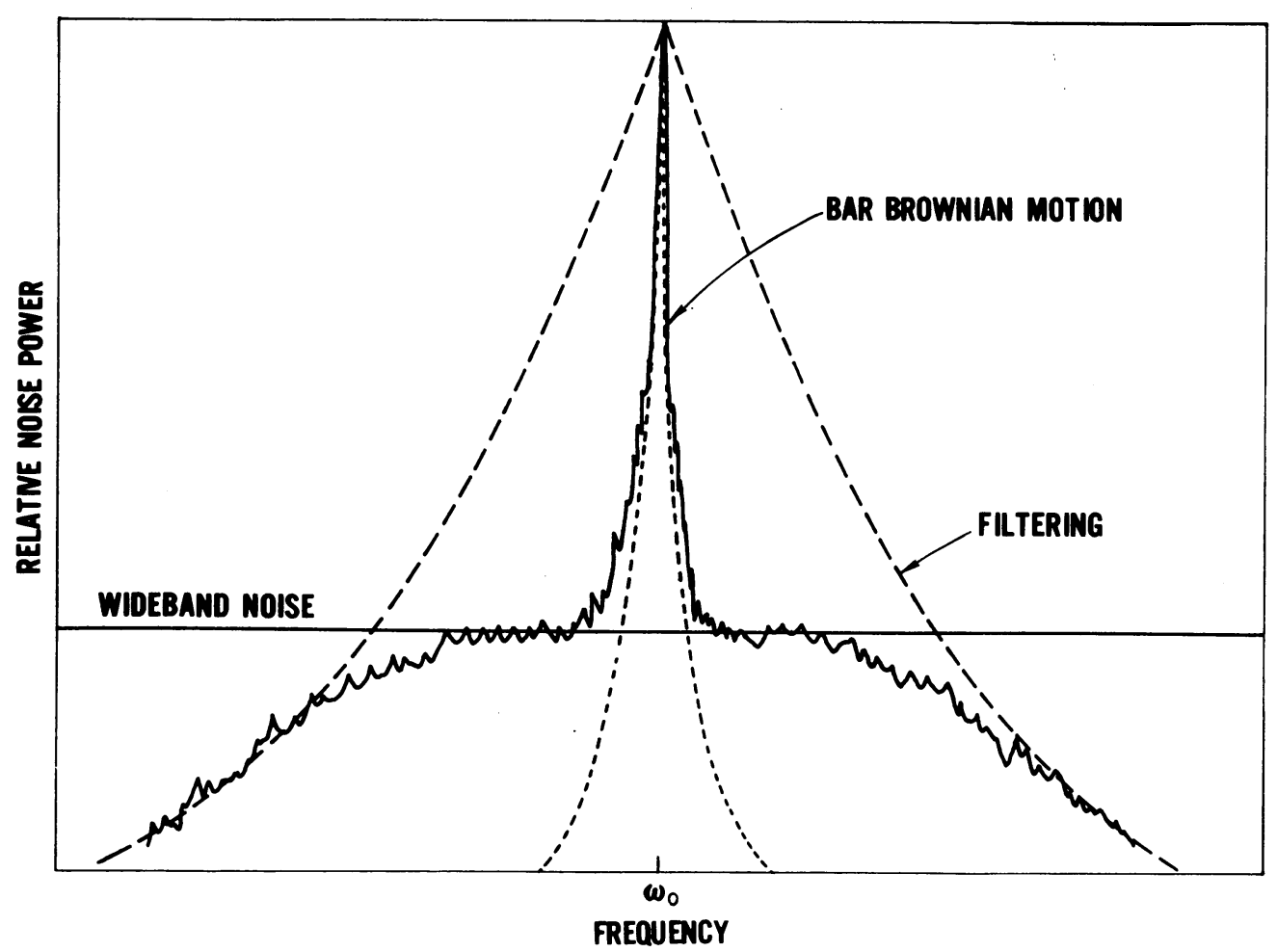

Fig. 3. A sketch of the spectral distribution of the antenna noise near the longitudinal resonance. The synchronous detector (demodulator) translates the peak in the spectrum to zero frequency. 
increase in sideband power and a relatively permanent change in power at resonance over a time equal to the Brownian motion autocorrelation time. The nonzero bandwidth of the Brownian motion power is due to unavoidable mechanical losses in the aluminum, rather than GR losses. Postdetection electrical low pass filtering eliminates wideband noise components at sideband frequencies higher than the Nyquist frequency corresponding to the data sampling rate. In most of the present GR antennas, the wideband noise plateau is more than a thousand times less than the Brownian motion power at the center of the Lorentzian line resonance.

The new GR antenna at Bell Labs is shown in Figure 4. The main transducers are mounted symmetrically about the center of the $3.6 \times 10^{6} \mathrm{gm}, 375 \mathrm{~cm}$ long aluminum bar. This symmetry cancels any sensitivity to bending modes which can couple to microseisms. Other modifications of the Weber detector include transducers on the

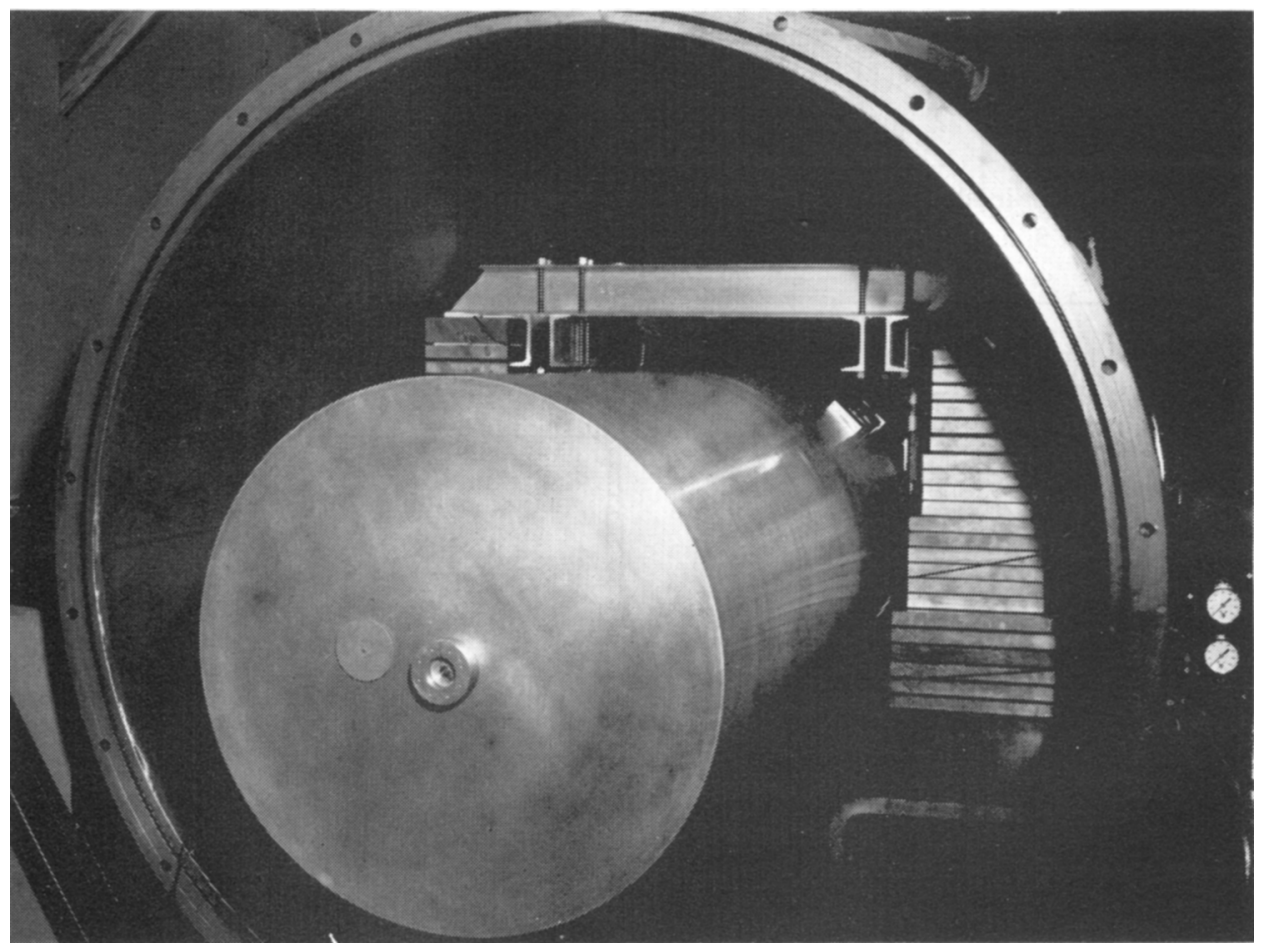

Fig. 4. Photograph of the new Bell Labs antenna with vacuum tank disassembled.

end, and low frequency air flotation of the entire vacuum tank assembly. The $710 \mathrm{~Hz}$ resonance of the bar is synchronously detected, and the $0.1 \mathrm{sec}$ averaged components $(X, Y)$ of the instantaneous antenna vector in the complex plane are sampled at a $10 \mathrm{~Hz}$ rate by a clock-tape data system. The amplitude of the bar oscillation (distance from the origin in the complex plane) is shown as a function of time in Figure 5. The slow change is Brownian motion of the bar (autocorrelation time $100 \mathrm{~s}$ ), whereas the 
fast, small amplitude noise is due primarily to the preamplifier. With this system, the total extraneous wideband noise power is sixteen times smaller than the Brownian motion power.

We wish to maximize our sensitivity to sudden changes in bar energy. Figure 6 shows the assumed signal signature and the expression for the total power signal to noise ratio for the detection of this signature. The noise denominator contains contributions from rapid components of the Brownian motion (proportional to the time resolution divided by the bar autocorrelation time), the preamplifier noises, and the transducer loss tangent, $\tan \delta$. If the preamplifier offset noise $R_{s}$ dominates the transducer loss noise, the maximum signal to noise ratio $(S / N)$ as a function of time resolu-

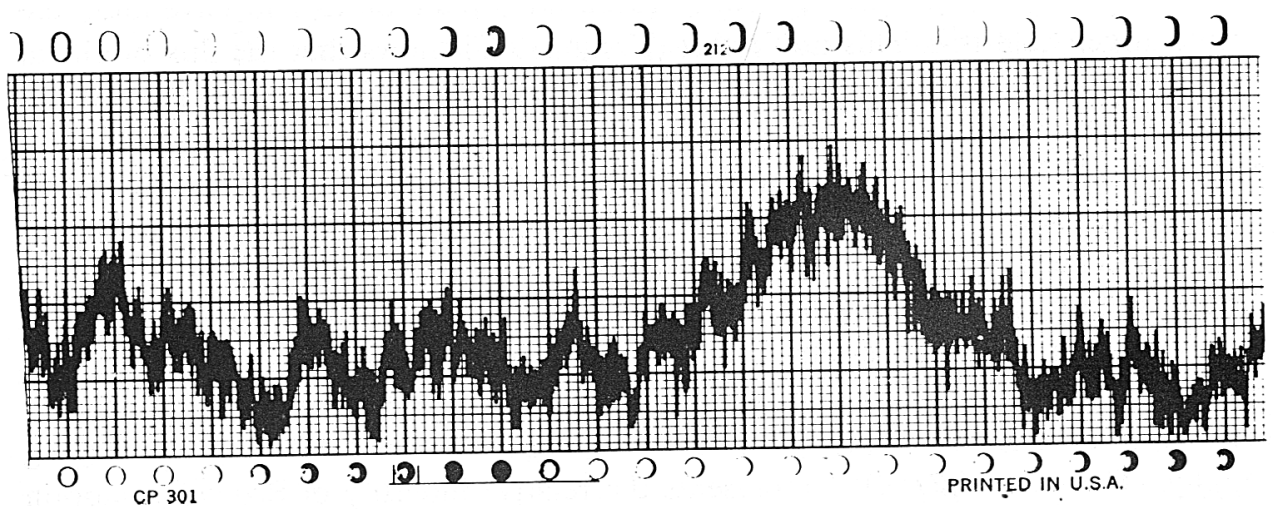

Fig. 5. Output amplitude of the Bell Labs antenna after demodulation. Slow changes are $k T$ noise from the bar, and fast noise is the preamplifier noise. Time scale: $30 \mathrm{~s}$ per small division.

$$
\begin{aligned}
& \frac{S}{N}=\frac{\frac{\beta}{2} \int \sigma(\gamma) d v}{k T \beta+/ 2 \tau+N / t} \quad+\ll \tau \\
& N=\frac{4}{\pi} k T\left(R_{s} C_{2}+\frac{\tan \zeta}{\omega}\right)+\cdots \\
& \left(\frac{S}{N}\right)_{\max } \sim m\left(\beta Q z_{x} / R_{s}\right)^{1 / 2}
\end{aligned}
$$

Fig. 6. Output amplitude signature due to a short burst of GR. If the wideband noises from the transducer loss and the preamplifier parallel noise are less than the preamplifier series noise from $R_{s}$, then the final expression for the power signal to noise ratio is obtained. 
tion is proportional to $m(\beta Q / N)^{1 / 2}$, where $Q=\omega \tau / 2$. We have maximized this for the new Bell Labs and U. Rochester antennas. There is some confusion regarding this notation. A $\frac{1}{4} k T$ event in our antenna would correspond to a $\frac{1}{40} k T$ event in an antenna of $\frac{1}{10}$ the mass and the same $\beta Q / N$. Quoting an event in fractions of $k T$ (the thermal noise of that antenna) does not imply a unique GR flux, unless $\beta Q / N$ and $m$ are known. A more sensitive way to search for GR is to look in the complex plane for a sudden change on the antenna vector. This is done in our computer program.

The number distribution for these sudden changes is shown as a function of square amplitude for $0.1 \mathrm{~s}$ time resolution in Figure 7. For $1 \mathrm{~s}$ resolution (longer integration), fewer than one $\frac{1}{2} k T$ (in the Bell Labs antenna) event per month would be expected for this system. We have carried out a search for three months and have found no events larger than this. This null result (Tyson, 1973) shows that the flux of GR at $710 \mathrm{~Hz}$ is considerably smaller now than Weber's claimed flux at $1660 \mathrm{~Hz}$ in 1970 . A $\frac{1}{2} k T$ excitation in the Bell Labs antenna would correspond to a GR flux which, if present at $1660 \mathrm{~Hz}$, would give Weber's antenna of 1970 an excitation of less than $\frac{1}{200} k T$. Because of occasional local interference, a more sensitive search may be carried out between two antennas in coincidence.

Several groups are now searching with two antennas in coincidence. The relative sensitivity assuming equal electronics noise is shown in Figure 8. These data are from preprints kindly supplied to us by the various groups. The electronics noise factor $N$ varies over a factor of ten among the various groups, but this technology is changing rapidly. (The factor $(\beta Q / N)^{1 / 2}$ represents the relative intensity of Brownian motion over wideband noise. The factor of bar mass $m$ comes from the integrated absorption cross section.) Note the large increase in sensitivity of Weber's experiment between 1970 and present. The present values of the electronics noise $N$ put Munich, Frascati, Paris, and Maryland roughly equal at the best sensitivity at $\sim 1660 \mathrm{~Hz}$, Moscow somewhat lower, and Bell Labs and U. Rochester nearly equal at $710 \mathrm{~Hz}$ at a sensitivity twice as high as any antennas at $1660 \mathrm{~Hz}$. If we had the combination of the Paris preamp and the Bell Labs bar and transducer, we would have a sensitivity six times higher. All the groups are presently improving their signal to noise, and we expect to see an order of magnitude increase in sensitivity within the next year. All these groups have so far indicated a null result for their search for GR, except Weber's group.

One possible explanation of this disparity may be in the kind of signature which is searched for. Most of the groups search in coincidence for a more or less sudden change in bar amplitude or phase, whereas Weber's current experiment searches in coincidence for fixed threshold crossings of a type of time derivative of the antenna vector: $\dot{X}^{2}+\dot{Y}^{2}$. Any momentary departure in relative phase or amplitude would yield a spike in this squared derivative, but would not be detected in the linear searches for GR performed by these other groups. In fact, Weber's best results (highest value of excess coincidences at zero time lag) seem to have been with nonlinear detection schemes. Since Weber's signal to noise ratio (real to random rate) has remained relatively constant during a greater than ten fold increase in sensitivity to GR, this suggests 
some other origin for these coincidences in the nonlinear detector. However, these important questions must be settled by direct experimentation. J. Weber has performed tests and has offered his help in the resolution of this problem. In collaboration with D. H. Douglass (University of Rochester) we are now recording $X, Y$, and $\dot{X}^{2}+\dot{Y}^{2}$ on digital magnetic tape at both the Bell Labs and Rochester antennas.

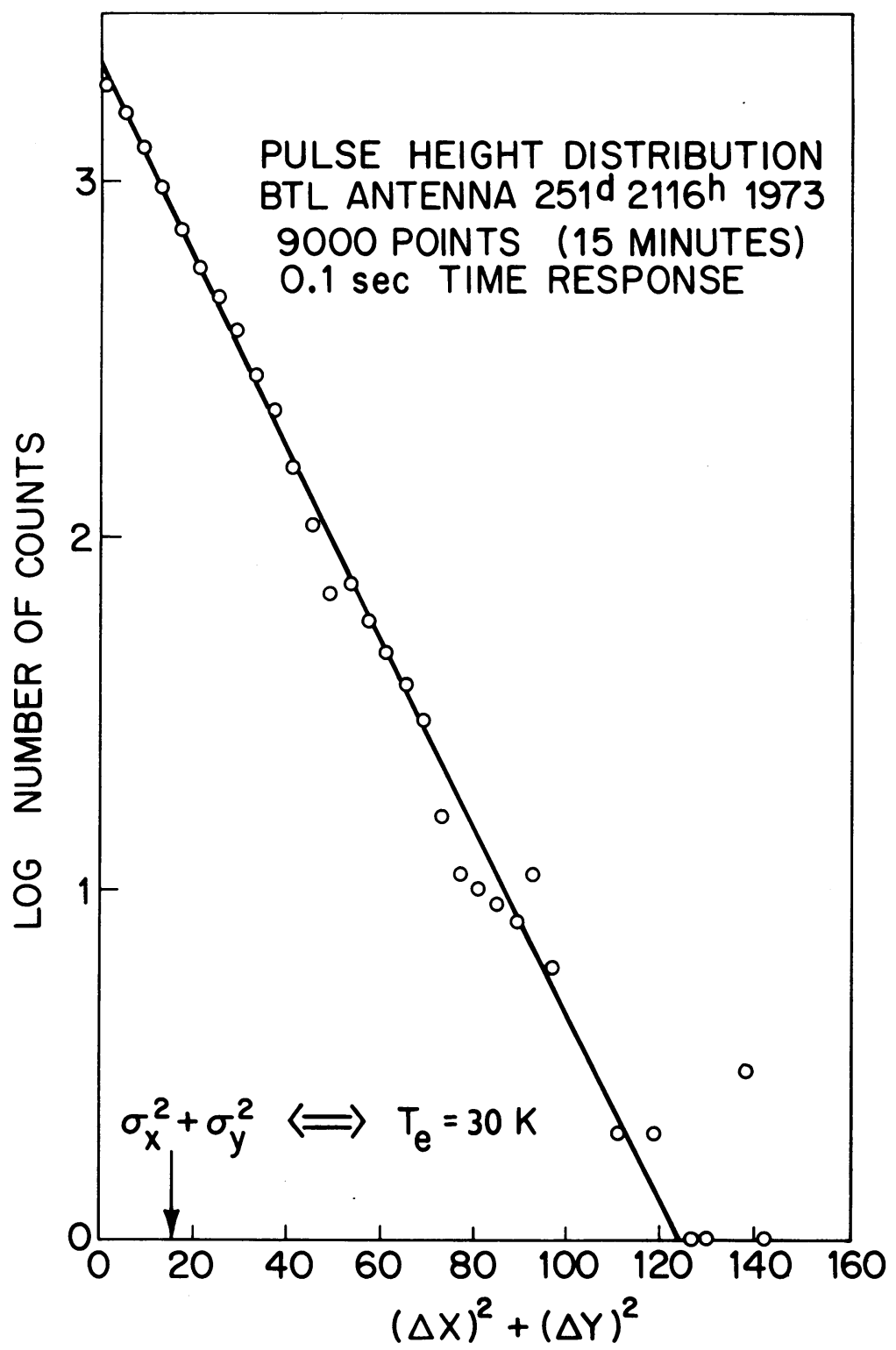

Fig. 7. Number distribution of sudden $(<0.1 \mathrm{~s})$ changes in amplitude or phase. Amplifier noise dominates. 


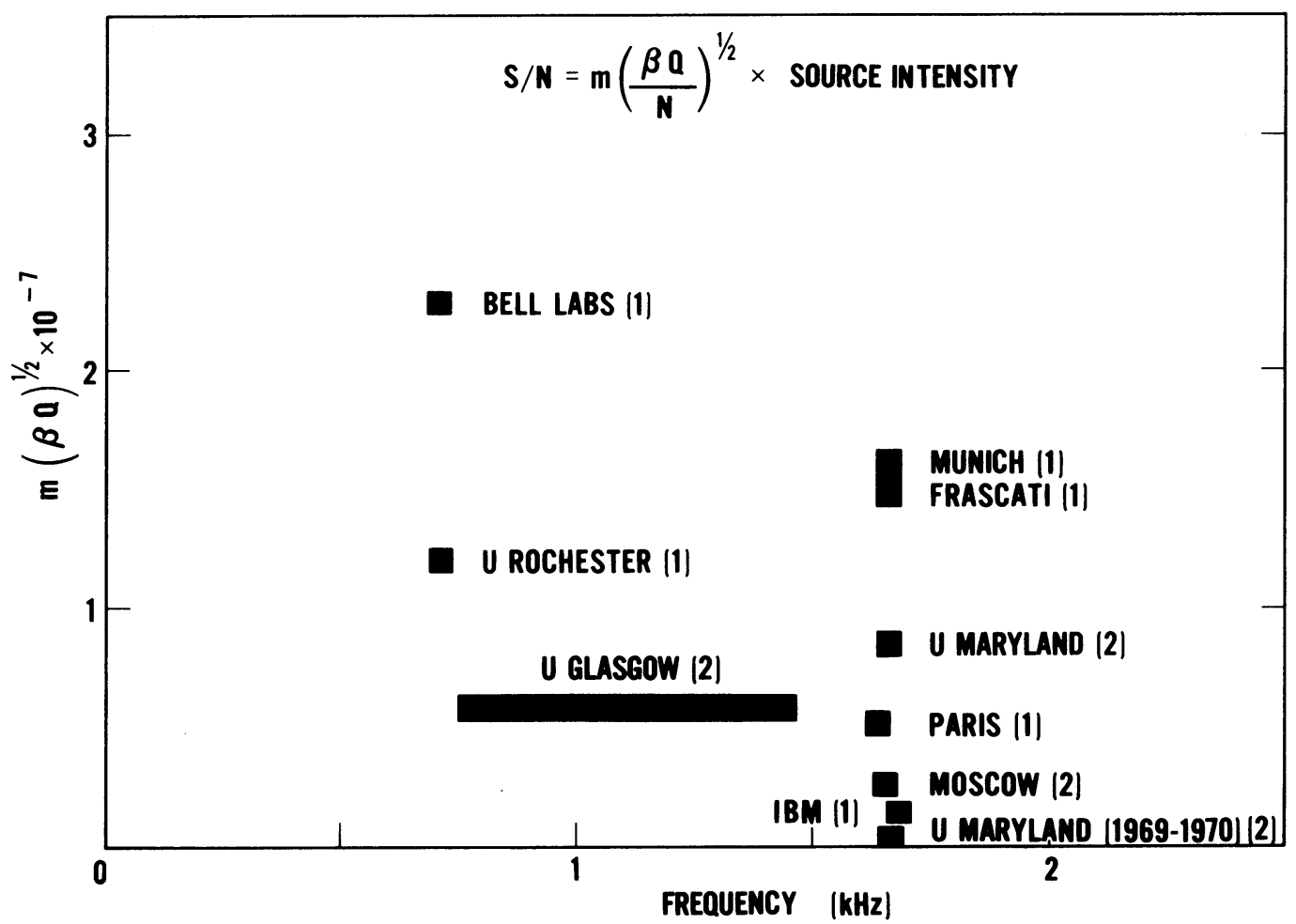

Fig. 8. Relative sensitivities to GR bursts of various antennas now operating, assuming equal amplifier noises and similar data analysis. Although the parameter $m(\beta Q)^{1 / 2}$ will not be easily changed, much better total sensitivity will be reached soon due to lower noise preamplifiers.

Various searches have been made for other types of radiation coincident with the reported Weber events, all with null results. (See Slusher and Tyson, 1973.) The limiting electro-magnetic flux per Weber event ranges between about $10^{-21} \mathrm{erg} \mathrm{cm}^{-2}$ $\mathrm{s}^{-1} \mathrm{~Hz}^{-1}$ for UHF, microwave, and infrared searches to $10^{-24} \mathrm{erg} \mathrm{cm}^{-2} \mathrm{~s}^{-1} \mathrm{~Hz}^{-1}$ for gamma rays. Some of these searches concentrated only on the galactic center region. In terms of total pulse energy, in erg $\mathrm{cm}^{-2}$ event ${ }^{-1}$, the positive and null GR observations are between $10^{4}$ and $10^{6}$, whereas electron neutrino null limits extend between $10^{2}$ and $10^{-7}$, muon neutrino limits $<10^{-4}$, gamma ray $<10^{-5}$, infrared $<10^{-9}$, and radio $<10^{-12}$. These upper limits put a severe constraint on any model of the possible source of large bursts of GR.

We now wish to present some preliminary results for the Bell Labs-U. Rochester two antenna coincidence experiment. The two antenna system has been operating at the design sensitivity (approx. 3 times Weber's present sensitivity) now for one month. Data are collected by totally independent tape systems, and these tapes are later correlated. Two independent analyses have been completed on part of this data, one by Bell Labs in collaboration with R. W. Lee (Stanford University) and one by the Rochester group. Both indicate no significant excess number of coincidences at zero time lag, with the two thresholds which we tried. These data are automatically 
calibrated in energy sensitivity and time by the introduction of artificial excitations of both antennas two seconds apart in real time, may times per week. Figure 9 shows the high threshold result. The ten standard deviation peak at $+2 \mathrm{~s}$ is the calibration peak, in this case at $1 k T$. This result implies that there were not more than about $10 \mathrm{GR}$ events imparting more than $\frac{1}{2} k T$ energy to these bars during this 16 days.

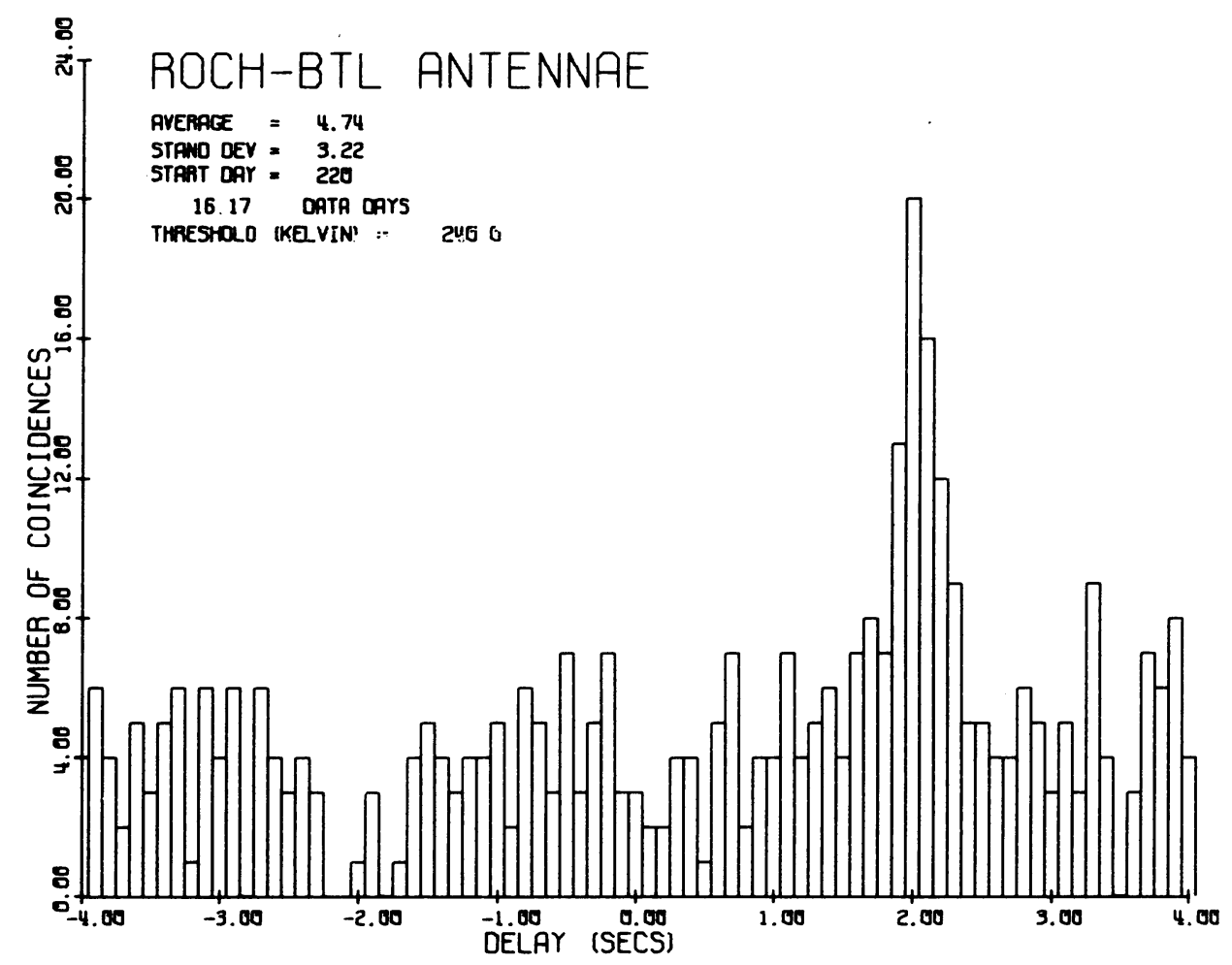

Fig. 9. Coincidence data for the Bell Labs-U. Rochester collaboration, using high threshold. No peak at zero lag is seen. The $10 \sigma$ peak at $+2 \mathrm{~s}$ lag is from 90 artificial excitations at a $1 k T$ level introduced simultaneous plus $2 \mathrm{~s}$.

Figure 10 shows the low threshold result in which 7006 and 4113 candidates were selected from each data tape by (as in Figure 9) convolving the raw amplitude and phase data with the time domain filter shown in Figure 11. This type of filter was also used during the last few years on our single antenna searches, and it has been very effective in eliminating sensitivity to occasional departures of amplitude or phase due to 'spikes' in the electronics. Returning to Figure 10, the $3 \sigma$ peak at $-3.8 \mathrm{~s}$ is not very significant, considering that there were 81 points in the lag plot with about 30 of them statistically independent. We are confident that there were few events above $\sim \frac{1}{4} k T$ $\left(\sim \frac{1}{10} k T\right.$ in Weber's bars) during this time.

We measure the efficiency of our detectors at various levels directly. For a $1 k T$ event, the Rochester antenna efficiency is $23 \%$ for \pm 50 ms resolution and $\sim 90 \%$ for 


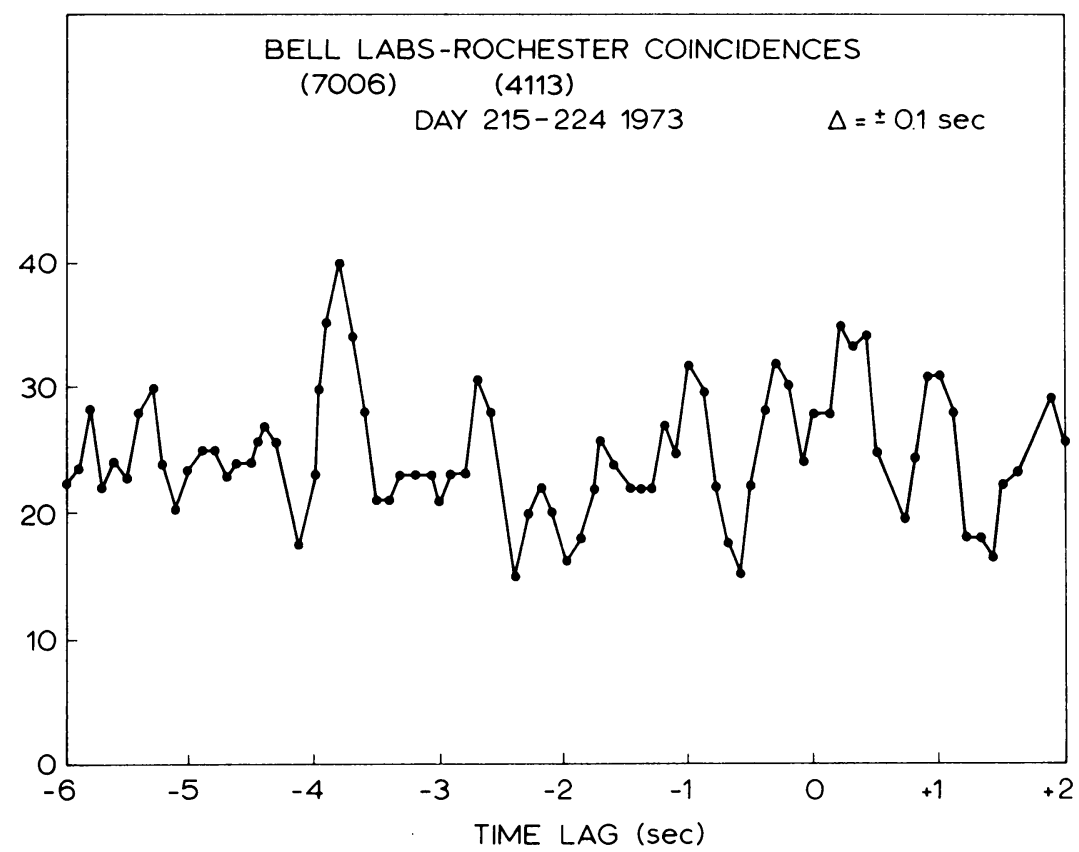

Fig. 10. Coincidence data for the Bell Labs-U. Rochester collaboration, using low threshold. No peak at zero lag is seen. No artificial calibration signals were applied.

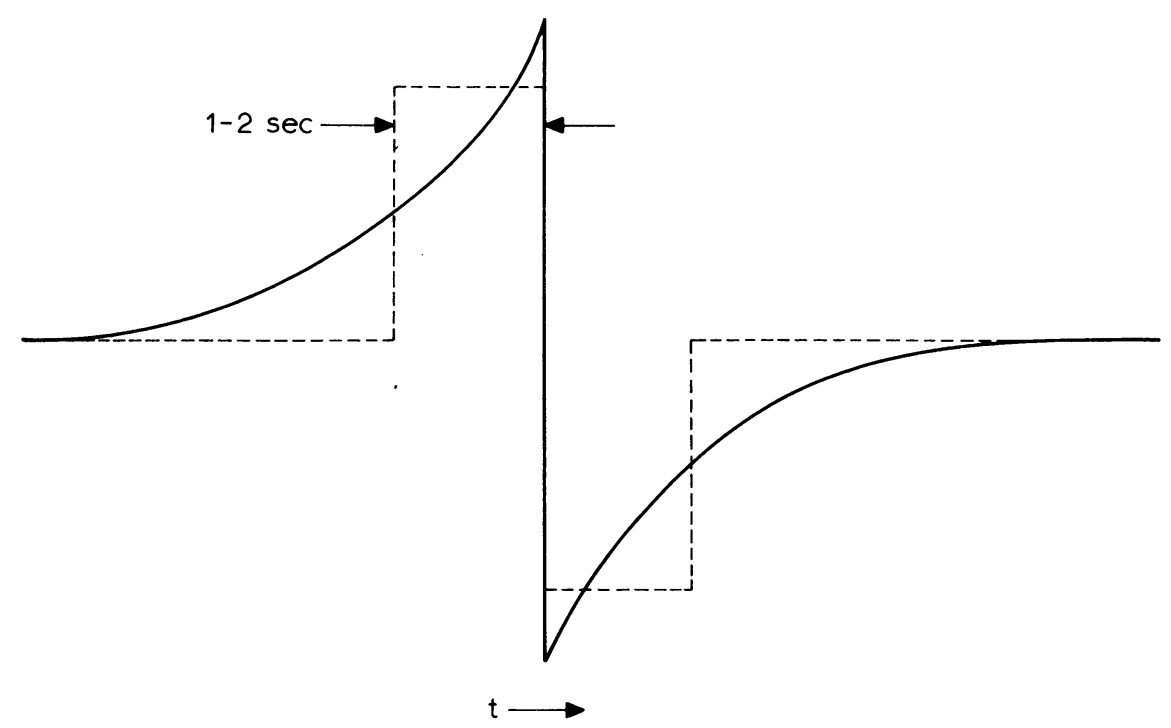

Fig. 11. Time-domain filter function used on magnetic tape data for the antenna coordinates $X$ and $Y$. This filter is nearly optimal for the signature in Figure 6, and it discriminates against any electrical spikes. 
$\pm 0.3 \mathrm{~s}$ resolution. The Bell Labs antenna is better. The efficiency of the two-antenna coincidence pair is $\sim 85 \%$ at $1 k T$ for $\pm 0.3 \mathrm{~s}$ resolution. At event intensities of $\frac{1}{4} k T$, the Rochester efficiency is $15 \%$ and the Bell Labs efficiency $33 \%$, for $\pm 0.3 \mathrm{~s}$ resolution At $\frac{1}{70} k T$, the Bell Labs efficiency is $\widetilde{<} \%$. Or course, for $\mathrm{GR}$, these antennas absorb $\sim 3$ times more energy from the wave, than Weber's antennas.

We emphasize that this experiment and Weber's experiment are sensitive to different signatures in the antenna amplitude or phase as a function of time; our analysis is sensitive to the kind of signature shown at the top of Figure 6, whereas Weber's current analysis is much more sensitive to 'delta functions' in the phase or amplitude as a function of time. The true origin of Weber's pulses remain an intriguing experimental problem worthy of our continued international effort.

The future holds possibilities for great improvements in sensitivity. We hope to push $\beta Q$ for our antennas from the present value of 40 up to $10^{4}$. Since the present calibrated noise temperature of our two antenna array is $20 \mathrm{~K}$, this increase in sensitivity would give noise temperatures less than $1 \mathrm{~K}$. We estimate that Weber's present antenna noise temperature is $\sim 200 \mathrm{~K}$, using his nonlinear algorithm. This implies that our antennas are $\sim 10$ times more sensitive to GR than Weber's present system, an increase of $>100$ over his system in 1970. But sensitivity increases of $>10^{3}$ may become possible with kilometer versions of the Hughes laser antenna (in space) or low-temperature designs. The suggestion of Burke (1973) may offer a unique output for a GR input. Prof. Braginsky will now suggest other possibilities.

\section{References}

Burke, W.: 1973, to be published in Phys. Rev.

Slusher, R. E. and Tyson, J. A.: 1973, Nature 243, 25.

Tyson, J. A.: 1973, Phys. Rev. Letters 31, 326.

Weber, J.: 1969, Phys. Rev. Letters 22, 1320.

Weber, J.: 1970a, Phys. Rev. Letters 24, 167.

Weber, J.: 1970b, Phys. Rev. Letters 25, 180.

Weber, J.: 1972, Nature 240, 28. 